\title{
Postcoronary pain and the postmyocardial infarction syndrome
}

\author{
R K T WILLIAMS,^ R E NAGLE,^ R A THOMPSON† \\ From the ${ }^{\star}$ Department of Cardiology, Selly Oak Hospital, Birmingham; and the †Regional Department of \\ Immunology, East Birmingham Hospital, Birmingham
}

SUMMARY Since non-specific chest pain, which is a common feature after myocardial infarction, may be due to a mild form of Dressler's (postmyocardial infarction) syndrome 80 patients were studied after confirmed myocardial infarction. Two patients had clinical features of classical Dressler's syndrome with high erythrocyte sedimentation rates and strongly positive results for antimyocardial antibodies. Twenty three patients had non-specific chest pain; none had clinical features of Dressler's syndrome. There was no difference in the erythrocyte sedimentation rate between this group and the remainder who had no pain. Equal numbers in each group had weakly positive test results for antimyocardial antibodies; none had a strongly positive result. Thus there appears to be no evidence for a mild form of Dressler's syndrome, and the erythrocyte sedimentation rate and weakly positive results for antimyocardial antibodies are of no diagnostic value in Dressler's syndrome.

William Dressler described the syndrome which bears his name in 1956 after observing fever, malaise, pleurisy, and pericarditis some weeks after myocardial infarction. ${ }^{1}$ These clinical features are often accompanied by a raised erythrocyte sedimentation rate, and his original idea that an immune process might underly the condition has been supported by the discovery in the blood of antibodies to cardiac muscle. ${ }^{23}$ The incidence of Dressler's syndrome has been about $3 \%$ in most studies ${ }^{4}$ including Dressler's own study of 44 patients. ${ }^{6}$ The postcardiotomy syndrome is generally considered to be an analogous condition after cardiac surgery.

We had found that many patients attending a coronary follow up clinic complained of sharp non-specific chest pain, which was unlike angina, but without other evidence of pericarditis or pleurisy. Many had a raised erythrocyte sedimentation rate. Such patients might have a mild form of Dressler's syndrome, and we therefore performed this prospective study of patients attending the follow up clinic to investigate the relation between symptoms, the erythrocyte sedimentation rate, and the presence of antimyocar-

Requests for reprints to Dr R K T Williams, Department of Geriatrics, Northwick Park Hospital, Watford Road, Harrow, Middlesex HA1 3UJ.

Accepted for publication 3 November 1983 dial antibodies, which is probably the most specific test for this condition.

\section{Patients and methods}

Eighty consecutive and unselected patients attending the coronary follow up clinic of a district general hospital were studied. They attended the clinic four to six weeks after acute myocardial infarction confirmed by sequential electrocardiographic changes and a characteristic rise in serum activity of cardiac enzymes (aspartate aminotransferase and hydroxybutyrate dehydrogenase) to more that twice the upper limit of normal. A full clinical history was taken and they were asked specifically about chest pain. Only those patients whose pain was non-anginal were included in the study. Clinical examination, chest radiography, and electrocardiography were performed. Blood was taken for estimation of the erythrocyte sedimentation rate and antimyocardial antibodies. Antimyocardial antibodies were detected by the indirect

Table Grading of the severity of myocardial infarction

\begin{tabular}{ll}
\hline Grade & Criteria \\
\hline 2: severe & $\begin{array}{c}\text { Abnormal cardiac impulse, third heart } \\
\text { sound, cardiomegaly and pulmonary } \\
\text { oedema on chest radiograph } \\
\text { Atrial sound, crepitations, pulmonary } \\
\text { venous congestion on chest radiograph } \\
\text { None of the above }\end{array}$ \\
0
\end{tabular}


immunofluorescence technique of Johnson and Dorling $^{7}$ using frozen sections of a heart post mortem from a subject with blood group 0 . Sera were screened initially at a dilution of $1 / 5$; the positive sera were subsequently titrated in doubling dilutions.

According to the severity of the infarction patients were graded into three groups during admission using the clinical criteria shown in the Table. ${ }^{8}$

Statistical tests of significance for difference between the groups were carried out using the MannWhitney $\mathrm{U}$ test for non-parametric samples.

\section{Results}

Of 80 patients, 11 had positive tests for antimyocardial antibodies; nine were weakly positive at a titre of $1 / 10$, and two strongly positive at a titre of $1 / 40$. These two patients were also considered clinically to have classical Dressler's syndrome and were therefore excluded from subsequent analysis. When the relation between non-anginal chest pain and a weakly positive result for antimyocardial antibodies was examined three of 23 patients with chest pain had weakly positive results and six of 55 patients who were pain free had weakly positive results.

The erythrocyte sedimentation rate in the group with weakly positive results for antimyocardial antibodies was compared with that in the group with negative results. The median rate for the weakly positive group was $14 \mathrm{~mm} / \mathrm{first}$ hour (range 1-63); the median rate for the negative group was $21 \mathrm{~mm} / \mathrm{first}$ hour (range 3-96). This difference was not statistically significant.

Twenty asymptomatic patients had erythrocyte sedimentation rates of more than 40 /first hour. The rates were also compared with the grade of severity in the preceding infarct. In 69 patients it was classified as mild, in 22 as moderate, and in 14 as severe. The rates for the mild group were compared with those for the moderate and severe groups combined. The median rate for the mild group was $11 \mathrm{~mm} /$ first hour (range 1-80); for the moderate and severe groups the median rate was $34 \mathrm{~mm} /$ first hour (range 3-120); this difference was highly significant $(\mathrm{p}<0.0003)$.

\section{Discussion}

The studies of Engle $e t a l^{9}$ and Trueman $e t a^{3}$ suggested that although low concentrations of antimyocardial antibodies are found in many conditions, they are only present at a high titre in Dressler's syndrome and the postcardiotomy syndrome. A test giving a strongly positive result is, therefore, likely to be the most specific available for these diseases. Two of our patients had antibodies at a high titre accompanied by a high erythrocyte sedimentation rate and very characteristic pain and malaise. We consider that these cases were examples of Dressler's syndrome and their number agrees with previous experience of the incidence of this condition (approximately $3 \%$ ).

Of the remaining 78 patients, 23 had chest pain, which could possibly represent a mild form of pericarditis or pleurisy. The proportion of symptomatic cases with a weakly positive antimyocardial antibodies result (3/23) was similar to that among patients who were pain free $(6 / 55)$. This suggests that a weakly positive result is of no clinical significance.

A raised erythrocyte sedimentation rate was not more common in patients with pain, and indeed 20 asymptomatic patients had rates of at least $40 \mathrm{~mm} / \mathrm{first}$ hour. Patients with more severe infarcts according to our clinical criteria had on average higher rates than did mild cases, but even some mild cases had abnormally raised rates. Most patients have a raised rate soon after myocardial infarction. The factors which determined the rate at the time of our follow up study six weeks later are not known, but the severity of muscle damage may be one of them. Clearly, a raised rate is of no help in the diagnosis of Dressler's syndrome.

The question of the true cause of non-specific sharp chest pain after myocardial infarction remains unanswered. Increased psychological sensitivity to mild skeletal pains, associated with a return to full activity after a period of immobilisation, seems a likely possibility. The symptoms often respond well to reassurance, explanation, and exercise.

This study tends to support the conventional view that Dressler's syndrome is an uncommon complication of acute myocardial infarction and makes it unlikely that the non-specific chest pain found so commonly during convalescence represents a mild form of it. A weakly positive antimyocardial antibody result test and a raised erythrocyte sedimentation rate are both quite common and of no diagnostic value in this connection.

A recent study by Welin $e t a l$, using the erythrocyte sedimentation rate as one of the diagnostic criteria, showed that the postmyocardial infarction syndrome tended to occur in larger infarcts. ${ }^{10}$ They also suggested that a positive antimyocardial antibody result might be of help in the diagnosis. We consider that our study provides further evidence that a strongly positive result is of use in diagnosing the postmyocardial infarction syndrome (possibly replacing the erythrocyte sedimentation rate as one of the diagnostic criteria).

\section{References}

1 Dressler W. A postmyocardial infarction syndrome: preliminary report of complication resembling idiopathic, 
recurrent, benign pericarditis. $\mathcal{F A M A} 1$ 1956; 160: 1379 83.

2 Van der Geld H. Anti-heart antibodies in the postpericardiotomy and the postmyocardial-infarction syndromes. Lancet 1964; ii: 617-21.

3 Trueman T, Thompson RA, Cummins P, Littler WA. Heart antibodies in cardiomyopathies. $\mathrm{Br}$ Heart $\mathcal{F}$ 1981; 46: 296-301.

4 Davidson C, Oliver MF, Robertson RF. Postmyocardial infarction syndrome. $\mathrm{Br} \mathrm{Med} \mathcal{F}$ 1961; ii: 535-9.

5 Shopfner CE. Post myocardial infarction syndrome. Radiology 1963; 81: 236-41.

6 Dressler W. The postmyocardial infarction syndrome. A report on 44 cases. Arch Intern Med 1959; 103: 28-42.
7 Johnson GD, Dorling J. Immunofluorescence and immunoperoxidase techniques. In: Thompson RA, ed. Techniques in clinical immunology. 2nd ed. Oxford and London: Blackwell Scientific Publications, 1981; 10637.

8 Nagle R, Gangola R, Picton-Robinson I. Factors influencing return to work after myocardial infarction. Lancet 1971; ii: 454-6.

9 Engle MA, McCabe JC, Ebert PA, Zabriskie J. The postpericardiotomy syndrome and antiheart antibodies. Circulation 1974; 49: 401-6.

10 Welin L, Vedin A, Wilhelmsson C. Characteristics, prevalence and prognosis of postmyocardial infarction syndrome. Br Heart F 1983; 50: 140-5. 